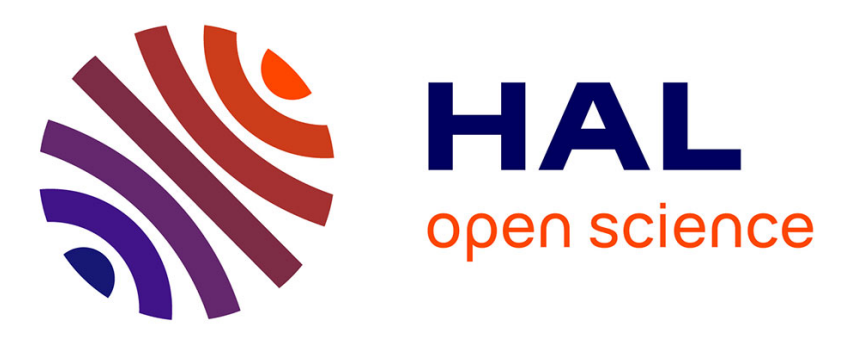

\title{
Adaptive fuzzy tracking control for a class of MIMO nonaffine uncertain systems
}

Abdesselem Boulkroune, Mohammed M'Saad, Mondher Farza

\section{To cite this version:}

Abdesselem Boulkroune, Mohammed M'Saad, Mondher Farza. Adaptive fuzzy tracking control for a class of MIMO nonaffine uncertain systems. Neurocomputing, 2012, 93, pp.Pages 48-55. 10.1016/j.neucom.2012.04.006 . hal-01061370

\section{HAL Id: hal-01061370 https://hal.science/hal-01061370}

Submitted on 10 Sep 2014

HAL is a multi-disciplinary open access archive for the deposit and dissemination of scientific research documents, whether they are published or not. The documents may come from teaching and research institutions in France or abroad, or from public or private research centers.
L'archive ouverte pluridisciplinaire HAL, est destinée au dépôt et à la diffusion de documents scientifiques de niveau recherche, publiés ou non, émanant des établissements d'enseignement et de recherche français ou étrangers, des laboratoires publics ou privés. 


\title{
Adaptive fuzzy tracking control for a class of MIMO nonaffine uncertain systems
}

\author{
A. Boulkroune ${ }^{\text {a,* }}$, M. M'Saad ${ }^{\text {b }}$, M. Farza ${ }^{\text {b }}$ \\ ${ }^{a}$ Department of Automatic, Faculty of Engineering Sciences, University of Jijel, BP. 98, OuledAissa, 18000 Jijel, Algeria \\ ${ }^{\mathrm{b}}$ GREYC, UMR 6072 CNRS, Université de Caen, ENSICAEN, 6 Bd, Maréchal Juin, 14050 Caen Cedex, France
}

\begin{abstract}
A B S T R A C T
In this paper, a novel fuzzy adaptive controller is investigated for a class of multi-input multi-output (MIMO) nonaffine systems with unknown control direction. An equivalent model in affine-like form is first derived for the original nonaffine system by using a Taylor series expansion. Then, a fuzzy adaptive control is designed based on the affine-like equivalent model. The adaptive fuzzy systems are used to appropriately approximate the unknown nonlinearities, while the lack of knowledge of the control direction being closely related to the sign of control gain matrix is handled by incorporating in the control law a Nussbaum-type function. A decomposition property of the control gain matrix is used in the controller design and the stability analysis. The effectiveness of the proposed fuzzy adaptive controller is illustrated through simulation results.
\end{abstract}

\section{Introduction}

Control process problems are more and more complex as the involved systems are multivariable in nature and exhibit uncertain nonlinear behaviors. This explains the fact that only few engineering solutions are available. Thanks to the universal approximation theorem [1], some adaptive fuzzy control systems [2-14] have been developed for a class of multivariable nonlinear uncertain systems. The stability of the underlying control systems has been investigated using a Lyapunov approach. The robustness issues with respect to the approximation error and external disturbances have been enhanced by appropriately modifying the available adaptive fuzzy controllers. The corner stone of such a modification consists in a robust compensator which is conceived using a sliding mode control design [3-5,7,9,11-14] or an $H^{\infty}$ based robust control design $[4,6,8,10]$. In the fuzzy indirect adaptive scheme $[2-5,8,10]$, the singularity problem occurring when determining the inverse of the estimated control gain matrix has been particularly solved thanks to a suitable projection inside the parameter space up to an priori knowledge on the system under control, namely a feasible set in which the singularity problem does not happen $[2,3,8,10]$. A genuine procedure involving an appropriate regularization of the estimated control

\footnotetext{
* Corresponding author.

E-mail addresses: boulkroune2002@yahoo.fr (A. Boulkroune) msaad@greyc.ensicaen.fr (M. M'Saad), mfarza@greyc.ensicaen.fr (M. Farza).
}

gain matrix has been also used in [4,5] up to an admissible tracking performance reduction. The key modeling assumption in these above fuzzy adaptive control schemes is that the systems considered are characterized by inputs appearing linearly in the system equation, i.e., the systems considered are affine-incontrol. To the authors' best knowledge, there are few works in the fuzzy control literature which were devoted to the control problem of the nonaffine multivariable systems.

In practice, there are many nonlinear systems with nonaffine structure, such as chemical reactors [15], biochemical process [16], some aircraft dynamics [17], dynamic model in pendulum control [18], etc. Some remarkable results for nonaffine monovariable systems have been obtained [19-31]. It is worth noting that affine systems are a special case of the nonaffine systems. Thus, all schemes in [19-31] can be applied directly to affine systems in which the control input appears in a linear fashion. In the literature, one can find five methods dealing with nonaffine problem, such as: (i) Method using Taylor series expansion in order to get an affine system (seen in $[19,20]$ ). (ii) Method using implicit function theorem (seen in [21-24]). (iii) Method exploiting the mean value theorem in order to obtain an affine form (seen in [25-28]). (iv) By differentiating the original system equation so that, in the augmented resulting model, the time derivative of the control input appears linearly and the latter can be used as a new control variable (seen in [29]). (v) By using a local inversion of the Takagi-Sugeno (TS) fuzzy affine model (seen in $[30,31]$ ). Note that there are two common modeling assumptions in these above adaptive control schemes [19-31] 
namely: the sign of the control-gain is known a priori and the model is monovariable. The sign of the control gain (i.e., the socalled control direction) represents motion direction of the system under any control, and knowledge of this sign makes adaptive control design much easier.

In the adaptive control literature, the unknown control-direction problem has been addressed by many methods: (i) By incorporating the Nussbaum-type function in the control law [32,33]. (ii) By directly estimating unknown parameters involved in the control direction [34]. (iii) By using the so-called correction-vector method for first order nonlinear systems [35]. (iv) Via a switching scheme based on a monitoring function for variable structure model reference adaptive control for linear plants [36]. (v) By incorporating a hysteresis-type function in adaptive fuzzy control $[37,38]$. (vi) By using a hysteresisdead-zone type function with the Nussbaum function in the adaptive control law [39]. Other hand, few results are available in the literature on the design of the fuzzy adaptive controllers for multivariable nonaffine nonlinear uncertain systems, [40-42]. Note that it is difficult and complicated to control this class of systems due to the existence of unknown nonaffine functions and the coupling strength among subsystems. In [40], this difficulty has been overcome by introducing some special type Lyapunov functions and taking advantage of the mean-value theorem, the backstepping design method and the approximation property of the fuzzy systems. In [41], the fuzzy adaptive controller designed for a class of multivariable nonaffine nonlinear systems has not been derived rigorously in mathematics. In fact, the time derivative of the Lyapunov function candidate is completely false. In [42], the considered systems are a more general class of nonaffine MIMO systems. To facilitate the controller design, the mean value theory is used to transform unknown non-affine functions into a structure which is similar to affine form. This control scheme has two main advantages: (i) it does not require a priori knowledge of the signs of the control gains and (ii) only one parameter is needed to be adjusted online in controller design procedure for each subsystem.

In this paper, a novel direct adaptive fuzzy controller is proposed for a class of uncertain multivariable nonaffine nonlinear systems with unknown control direction. Note that the approach proposed in this paper can be considered as an extension of our previous works $[11,13]$. The main contributions of this paper are emphasized below:

(i) To the authors' best knowledge, in the literature, there are no results reported on the direct fuzzy adaptive control design for our considered class of the non-affine multivariable systems with unknown control direction (i.e., system (1)).

(ii) A unique Nussbaum-type function is used in order to estimate the true control direction.

(iii) The combined effect of the fuzzy approximation error together with the higher order terms (HOT) issued from the use of the Taylor series expansion is compensated for by a dynamical robust adaptive control term.

\section{Problem statement, preliminaries and fuzzy systems}

\subsection{Problem statement and preliminaries}

Consider the following class of MIMO nonaffine nonlinear uncertain systems:

$y_{1}^{\left(r_{1}\right)}=f_{1}(x, u)+\Lambda_{1}(t)$

$y_{p}^{\left(r_{p}\right)}=f_{p}(x, u)+\Lambda_{p}(t)$ where $x=\left[x_{1}^{T}, \ldots, x_{p}^{T}\right]^{T} \in R^{r}$ is the overall state vector which is assumed available for measurement, where $x_{i}=\left[y_{i}, \dot{y}_{i}, \ldots\right.$, $\left.y_{i}^{\left(r_{i}-1\right)}\right]^{T} \in R^{r_{i}}, \forall i=1, \ldots, p$ and $r_{1}+\cdots+r_{p}=r . u=\left[u_{1}, \ldots, u_{p}\right]^{T} \in R^{p}$ is the control input vector, and $f_{i}(x, u), i=1, \ldots, p$ are smooth unknown nonaffine nonlinear functions. $\Lambda_{i}(t), \quad i=1, \ldots, p$ are unknown bounded disturbances.

Let us also denote

$y^{(r)}=\left[y_{1}^{\left(r_{1}\right)}, \ldots, y_{p}^{\left(r_{p}\right)}\right]^{T}, \quad F(x, u)=\left[f_{1}(x, u), \ldots, f_{p}(x, u)\right]^{T}$, $\Lambda(t)=\left[\Lambda_{1}(t), \ldots, \Lambda_{p}(t)\right]^{T}$. form:

Then, the system (1) can be rewritten in the following compact

$y^{(r)}=F(x, u)+\Lambda(t)$

Throughout this paper, one makes the following realistic assumptions:

Assumption 1. The desired trajectory vector $x_{d}=\left[x_{d 1}^{T}, \ldots, x_{d p}^{T}\right.$, $\left.y_{d 1}^{\left(r_{1}\right)}, \ldots, y_{d p}^{\left(r_{p}\right)}\right]^{T}$, where $x_{d i}=\left[y_{d i}, \dot{y}_{d i}, \ldots, y_{d i}^{\left(r_{i}-1\right)}\right]^{T}, \forall i=1, \ldots, p$, is supposed to be continuous, bounded and available for measurement. Then, one has $x_{d} \in \Omega_{x_{d}} \subset R^{r+p}$, with $\Omega_{x_{d}}$ is a known compact set.

Assumption 2. The matrix $\partial F(x, u) / \partial u$ is non-singular. Its sign is assumed to be unknown. But, it is must be positive-definite or negative-definite.

Remark 1. Assumption 2 ensures that the matrix $\partial F(x, u) / \partial u$ is always regular. This assumption can be seen as a controllability condition and is not restrictive as it is satisfied by many affine or nonaffine (MIMO or SISO) physical systems: e.g., robotic systems, induction motors, chaotic systems, some mechanical systems. Note that the affine nonlinear systems are a special case of the non-affine systems. In the affine SISO case, this assumption is very standard (see [31,32], and reference therein) and is equivalent to a control gain which should be strictly positive or strictly negative. In the affine MIMO case, it is equivalent to a control gains matrix which should be non-singular (see [2-14]).

The objective is to design a continuous adaptive control law $u_{i}$ (for all $i=1, \ldots, p$ ) which ensures that the state vector $x_{i}$ asymptotically tracks a time-varying desired trajectory $x_{d i}$, while all the signals in the derived closed-loop system remain bounded despite uncertainties and bounded external disturbances. To quantify this objective, the tracking errors are defined as follows:

$e_{1}=y_{d 1}-y_{1}$
$\vdots$
$e_{p}=y_{d p}-y_{p}$

Let us define also the filtered tracking error as

$S=\left[S_{1}, \ldots, S_{p}\right]^{T}$

with

$S_{i}=\left[\frac{d}{d t}+\lambda_{i}\right]^{r_{i}-1} e_{i}, \quad$ for $\quad \lambda_{i}>0 \quad \forall i=1, \ldots, p$.

Remark 2. Notice that if one chooses $\lambda_{i}>0$, with $i=1, \ldots, p$, then the roots of the polynomial $H_{i}(s)=\lambda_{i}^{r_{i}-1}+\left(r_{i}-1\right) \lambda_{i}^{r_{i}-2} s+\cdots+$ $\left(r_{i}-1\right) \lambda_{i} s^{r_{i}-2}+s^{r_{i}-1}$ related to the characteristic equation of $S_{i}=0$ are all in the open left-half plane. From (5), it follows that $e_{i} \rightarrow 0$ asymptotically as $S_{i} \rightarrow 0$. Thus, the problem of tracking the $r_{i}$-dimensional vector $y_{d i}$ can be replaced by a 1 st-order stabilization problem in the scalar $S_{i}$.

In this case, the control objective becomes the design of a controller to force $S_{i} \rightarrow 0$ asymptotically, for $i=1, \ldots, p$. 
The time derivative of $S_{i}$, for $i=1, \ldots, p$, can be written as

$\dot{S}_{i}=v_{i}-f_{i}(x, u)-\Lambda_{i}(t)$, for $i=1, \ldots, p$

with

$v_{i}=y_{d i}^{\left(r_{i}\right)}+\beta_{i, r_{i}-1} e_{i}^{\left(r_{i}-1\right)}+\cdots+\beta_{i, 1} \dot{e}_{i}, \quad$ for $\quad i=1, \ldots, p$

where

$\beta_{i, j}=\frac{\left(r_{i}-1\right) !}{\left(r_{i}-j\right) !(j-1) !} \lambda_{i}^{r_{i}-j}, \quad$ for $\quad i=1, \ldots, p, \quad j=1, \ldots, r_{i}-1$

denote $V=\left[v_{1}, \ldots, v_{p}\right]^{T}$, then (6) can be written in the following compact form

$\dot{S}=V-F(x, u)-\Lambda(t)$.

Now, to facilitate the control system design for the system (2), one transforms the nonaffine system (2) into an affine system by performing a Taylor series expansion around an unknown optimal control $u=u^{*}(x)$ as follows:

$F(x, u)=F(x)+G(x) u+H(x, u)$

with

$F(x)=\left[F_{1}(x), \ldots, F_{p}(x)\right]^{T}=F\left(x, u^{*}(x)\right)-[\partial F(x, u) / \partial u]_{u=u^{*}(x)} u^{*}(x)$,

$G(x)=\left[g_{i j}(x)\right]=[\partial F(x, u) / \partial u]_{u=u^{*}(x)}$,

where $H(x, u)$ is the higher order terms (HOT) of the expansion, and $u=u^{*}(x)$ is an unknown smooth function minimizing the HOT.

Motivated by [11-14,43-46] and since the matrix $G(x)$ is not generally symmetric, the following important lemma will be exploited in the controller design and stability analysis.

Lemma 1. $[43,44]$ Any real matrix $G(x) \in R^{P \times P}$ with non-zero leading principal minors can be decomposed as follows:

$G(x)=G_{s}(x) D T(x)$

where $G_{S}(x) \in R^{P \times P}$ is a symmetric positive-definite matrix, $D \in R^{P \times P}$ is a diagonal matrix with +1 or -1 on the diagonal, and $T(x) \in R^{P \times P}$ is a unity upper triangular matrix. The diagonal elements of $D$ are nothing else than the ratios of the signs of the leading principal minors of $G(x)$.

Remark 3. Note that if $G(x)$ has non-zero leading principal minors, three cases can arise:

- If $G(x)$ is positive-definite, then $D=I_{P}$

- If $G(x)$ is negative-definite, then $D=-I_{p}$.

- While in the case where $G(x)$ is indefinite, the matrix $D$ has +1 and -1 on its principal diagonal.

In order to cope with the unknown control direction (i.e., the unknown sign of the control gain matrix $G(x)$ ), the Nussbaum gain technique will be used in Section 3.

Properties of a Nussbaum-function: A function $N(\zeta)$ is called a Nussbaum-function, if it has the following useful properties [47,48]:

(1) $\lim _{s \rightarrow+\infty} \sup \frac{1}{s} \int_{0}^{s} N(\zeta) d \zeta=+\infty$
(2) $\lim _{s \rightarrow+\infty} \inf \frac{1}{s} \int_{0}^{s} N(\zeta) d \zeta=-\infty$

Example: The following functions are Nussbaum functions [47-49]:

$N_{1}(\zeta)=\zeta^{2} \cos (\zeta), \quad N_{2}(\zeta)=\zeta \cos (\sqrt{|\zeta|})$, and $\quad N_{3}(\zeta)=\cos \left(\frac{\pi}{2} \zeta\right) e^{\zeta^{2}}$, of course, the cosine in the above examples can be replaced by the sine. It is very easy to show that $N_{1}(\zeta), N_{2}(\zeta)$, and $N_{3}(\zeta)$ are Nussbaum functions. For clarity, the even Nussbaum $N(\zeta)=\zeta^{2} \cos (\zeta)$ will be used throughout this paper.

Thereafter, one needs the following lemma in the stability analysis.

Lemma 2. $[47,50]$ Let $V($.$) and \zeta($.$) be smooth functions defined on$ $\left[0, t_{f}\right)$, with $V(t) \geq 0, \forall t \in\left[0, t_{f}\right)$, and $N($.$) be an even Nussbaum$ function. If the following inequality holds:

$V(t) \leq c_{0}+\int_{0}^{t}(d N(\zeta)+1) \dot{\zeta} d \tau, \quad \forall t \in\left[0, t_{f}\right)$,

where $d$ is non-zero constant and $c_{0}$ represents some suitable constant, then $V(t), \zeta(t)$ and $\int_{0}^{t}(d N(\zeta)+1) \dot{\zeta} d \tau$ must be bounded on $\left[0, t_{f}\right)$.

Proof of Lemma 2. See the proof in [47].

\subsection{Dynamics of the filtered tracking errors}

Using the matrix decomposition (10) and the expression (9), the dynamics (8) can be rewritten as follows:

$\dot{S}=V-F(x)-G_{s}(x) D T(x) u-H(x, u)-\Lambda(t)$.

Now let us consider the following realistic assumption.

Assumption 3. The matrix $G(x)$ is of class $C^{1}$ and satisfies the following property:

$\partial g_{i j}(x) / \partial y_{i}^{\left(r_{i}-1\right)}=0, \quad \forall i=1,2, \ldots, p \quad$ and $\quad j=1,2, \ldots, p$.

\section{Remark 4.}

a) Although Assumption 3 restricts the considered class of MIMO nonaffine systems, many affine physical systems, such as robotic systems and electric machines, fulfill such a property [11-14].

b) The required property on the partial derivatives of the control gain matrix ensures that the time derivative of $G_{s}^{-1}(x)$ depends only on the state vector $x$ (i.e., it ensures that $d G_{s}^{-1}(x) / d t$ does not depend on the system inputs) [11-14].

From Eq. (12), one has

$G_{s}^{-1}(x) \dot{S}=G_{s}^{-1}(x)[V-F(x)]-D T(x) u-G_{s}^{-1}(x) H(x, u)-G_{s}^{-1}(x) \Lambda(t)$

Posing $G_{1}(x)=G_{s}^{-1}(x), F_{1}(x, u)=G_{s}^{-1}(x)[V-F(x)]-[D T(x)-D] u$, and $H_{1}(x, u, \Lambda)=G_{s}^{-1}(x)(H(x, u)+\Lambda(t))$, Eq. (13) becomes

$G_{1}(x) \dot{S}=F_{1}(x, u)-D u-H_{1}(x, u, \Lambda)$

One can rearrange (14) as follows

$G_{1}(x) \dot{S}+\frac{1}{2} \dot{G}_{1} S=\alpha(z)-D u-H_{1}(x, u, \Lambda)$

where $\quad \alpha(z)=\left[\alpha_{1}\left(z_{1}\right), \ldots, \alpha_{p}\left(z_{P}\right)\right]^{T}=F_{1}(x, u)+\frac{1}{2} \dot{G}_{1}(x) S \quad$ with $z=\left[z_{1}^{T}, z_{2}^{T}, \ldots, z_{p}^{T}\right]^{T}$. By examining the expressions of $F_{1}(x, u)$, the vectors $z_{i}$ can be determined as follows:

$z_{1}=\left[x^{T}, u_{2}, \ldots, u_{p}\right]^{T}$

$z_{2}=\left[x^{T}, u_{3}, \ldots, u_{p}\right]^{T}$

$z_{p-1}=\left[x^{T}, u_{p}\right]^{T}$

$z_{p}=x$

It is clear from the property of the matrix of $D T(x)-D$, that $z_{1}$ depends on control inputs $u_{2}, \ldots, u_{p}, z_{2}$ depends on $u_{3}, \ldots, u_{p}$, and 
so on. In fact, the structure of the nonlinearities $\alpha(z)$ is known under the name "upper triangular control structure". Recall that this useful structure allows for algebraic loop free sequential synthesis of control signals $u_{i}, \forall i=1,2, \ldots, p$.

Define the operating compact sets as follows:

$\Omega_{z_{i}}=\left\{\left[x^{T}, u_{i+1}, \ldots, u_{p}\right]^{T} \mid x \in \Omega_{x} \subset R^{r}, x_{d} \in \Omega_{x_{d}}\right\}, \quad i=1, \ldots, p-1$,

$\Omega_{z_{p}}=\left\{x \mid x \in \Omega_{x} \subset R^{r}\right\}$.

Remark 5. Since the nonlinear functions $\left(\alpha(z)\right.$ and $\left.H_{1}(x, u, A)\right)$ and the matrix $D$ are unknown, moreover $H_{1}(x, u, \Lambda)$ depends explicitly on the input $u$, the control system design to asymptotically stabilize the dynamics (15) is very difficult. Thereafter, to overcome such problems, one will use

- an adaptive fuzzy system to approximate the unknown nonlinear function $\alpha(z)$,

- a Nussbaum function to estimate the sign of the matrix $D$ (i.e., the sign of the control gain matrix $G(x))$ and,

- a dynamic adaptive robust control to dynamically compensate for the effect of the uncertain nonlinearity $H_{1}(x, u, \Lambda)$.

\subsection{Description of the fuzzy logic system}

The basic configuration of a fuzzy logic system consists of a fuzzifier, some fuzzy IF-THEN rules, a fuzzy inference engine and a defuzzifier, as shown in Fig. 1. The fuzzy inference engine uses the fuzzy IF-THEN rules to perform a mapping from an input vector $\underline{x}^{T}=\left[x_{1}, x_{2}, \ldots, x_{n}\right] \in R^{n}$ to an output $\hat{f} \in R$. The $i$ th fuzzy rule is written as

$R^{(i)}:$ if $x_{1}$ is $A_{1}^{i}$ and $\ldots$ and $x_{n}$ is $A_{n}^{i}$ then $\hat{f}$ is $f^{i}$

where $A_{1}^{i}, A_{2}^{i}, \ldots$, and $A_{n}^{i}$ are fuzzy sets and $f^{i}$ is the fuzzy singleton for the output in the ith rule. By using the singleton fuzzifier, product inference, and center-average defuzzifier, the output of the fuzzy system can be expressed as follows:

$\hat{f}(\underline{x})=\frac{\sum_{i=1}^{m} f^{i}\left(\prod_{j=1}^{n} \mu_{A_{j}^{i}}\left(x_{j}\right)\right)}{\sum_{i=1}^{m}\left(\prod_{j=1}^{n} \mu_{A_{j}^{i}}\left(x_{j}\right)\right)}=\theta^{T} \psi(\underline{x})$

where $\mu_{A_{j}^{i}}\left(x_{j}\right)$ is the degree of membership of $x_{j}$ to $A_{j}^{i}, m$ is the number of fuzzy rules, $\theta^{T}=\left[f^{1}, f^{2}, \ldots, f^{m}\right]$ is the adjustable parameter vector (composed of consequent parameters), and $\psi^{T}=\left[\psi^{1} \psi^{2} \ldots \psi^{m}\right]$ with

$\psi^{i}(\underline{x})=\frac{\left(\prod_{j=1}^{n} \mu_{A_{j}^{i}}\left(x_{j}\right)\right)}{\sum_{i=1}^{m}\left(\prod_{j=1}^{n} \mu_{A_{j}^{i}}\left(x_{j}\right)\right)}$

being the fuzzy basis function (FBF). Throughout the paper, it is assumed that the FBFs are selected so that there is always at least one active rule [1], i.e., $\Sigma_{i=1}^{m}\left(\Pi_{j=1}^{n} \mu_{A_{j}^{i}}\left(x_{j}\right)\right)>0$.

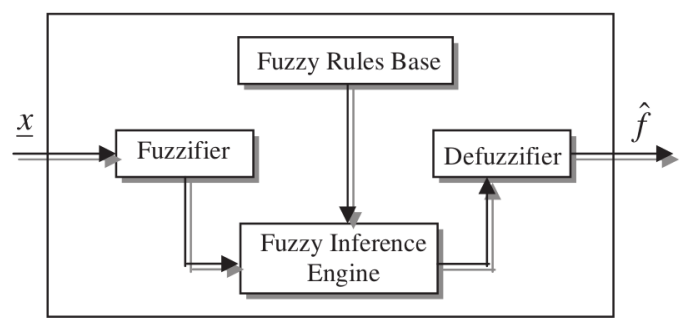

Fig. 1. Basic configuration of a fuzzy logic system.
It is worth noting that the fuzzy system (19) is commonly used in control applications. Following the universal approximation results, the fuzzy system (19) is able to approximate any nonlinear smooth function $f(x)$ on a compact operating space to an arbitrary degree of accuracy [1]. Of particular importance, it is assumed that the structure of the fuzzy system, namely the pertinent inputs, the number of membership functions for each input and the number of rules, and the membership function parameters are properly specified beforehand. The consequent parameters are then determined by appropriate parameter adaptation algorithms.

\section{Design of fuzzy adaptive controller}

The unknown nonlinear function $\alpha_{i}\left(z_{i}\right)$ in (15) can be approximated, on the compact set $\Omega_{z_{i}}$, by the linearly parameterized fuzzy systems (19) as follows:

$\hat{\alpha}_{i}\left(z_{i}, \theta_{i}\right)=\theta_{i}^{T} \psi_{i}\left(z_{i}\right), \quad i=1, \ldots, p$,

where $\psi_{i}\left(z_{i}\right)$ is the fuzzy basis function (FBF) vector, which is fixed a priori by the designer, and $\theta_{i}$ is the adjustable parameter vector of the fuzzy system.

Let us define

$\theta_{i}^{*}=\arg \min \left[\sup _{z_{i} \in \Omega_{z_{i}}}\left|\alpha_{i}\left(z_{i}\right)-\hat{\alpha}_{i}\left(z_{i}, \theta_{i}\right)\right|\right]$

as the optimal value of $\theta_{i}$ which is mainly introduced for analysis purposes as its value is not needed when implementing the controller.

Define

$\tilde{\theta}_{i}=\theta_{i}-\theta_{i}^{*} \quad$ and $\quad \varepsilon_{i}\left(z_{i}\right)=\alpha_{i}\left(z_{i}\right)-\hat{\alpha}_{i}\left(z_{i}, \theta_{i}^{*}\right) \quad$ with $\quad \hat{\alpha}_{i}\left(z_{i}, \theta_{i}^{*}\right)=\theta_{i}^{* T} \psi_{i}\left(z_{i}\right)$

as the parameter estimation error and the fuzzy approximation error, respectively.

As in the literature $[1-14,20,21,27,28,30-33,50-58]$, one assumes that the used fuzzy systems do not violate the universal approximator property on the compact set $\Omega_{z_{i}}$, which is assumed large enough so that the input vector of those fuzzy systems remains in $\Omega_{z_{i}}$ under the closed-loop control system. It is hence reasonable to assume that the fuzzy approximation error is bounded for all $z_{i} \in \Omega_{z_{i}}$, i.e.,

$\left|\varepsilon_{i}\left(z_{i}\right)\right| \leq \bar{\varepsilon}_{i}, \quad \forall z_{i} \in \Omega_{z_{i}}$,

where $\bar{\varepsilon}_{i}$ is an unknown constant.

Now, let us denote

$\hat{\alpha}(z, \theta)=\left[\hat{\alpha}_{1}\left(z_{1}, \theta_{1}\right), \ldots, \hat{\alpha}_{p}\left(z_{p}, \theta_{p}\right)\right]^{T}=\left[\theta_{1}^{T} \psi_{1}\left(z_{1}\right), \ldots, \theta_{p}^{T} \psi_{p}\left(z_{p}\right)\right]^{T}$,

$\varepsilon(z)=\left[\varepsilon_{1}\left(z_{1}\right), \ldots, \varepsilon_{p}\left(z_{p}\right)\right]^{T}$,

$\bar{\varepsilon}=\left[\bar{\varepsilon}_{1}, \ldots, \bar{\varepsilon}_{p}\right]^{T}$.

From the above analysis, one has

$\hat{\alpha}(z, \theta)-\alpha(z)=\hat{\alpha}(z, \theta)-\hat{\alpha}\left(\bar{z}, \theta^{*}\right)+\hat{\alpha}\left(\bar{z}, \theta^{*}\right)-\alpha(z)$,

$$
=\hat{\alpha}(z, \theta)-\hat{\alpha}\left(z, \theta^{*}\right)-\varepsilon(z) \text {, }
$$$$
=\tilde{\theta}^{T} \psi(z)-\varepsilon(z) \text {. }
$$

where $\tilde{\theta}^{T} \psi(z)=\left[\tilde{\theta}_{1}^{T} \psi_{1}\left(z_{1}\right), \ldots, \tilde{\theta}_{p}^{T} \psi_{p}\left(z_{p}\right)\right]^{T}$, and $\tilde{\theta}_{i}=\theta_{i}-\theta_{i}^{*}$, for $i=1, \ldots, p$.

From (24), one can rewrite (15) as follows:

$$
\begin{aligned}
\frac{d}{d t}\left[\frac{1}{2} S^{T} G_{1}(x) S\right] & =S^{T} \alpha(z)-S^{T} D u-S^{T} H_{1}(x, u, \Lambda) \\
& =-S^{T} \tilde{\theta}^{T} \psi(z)+S^{T} \varepsilon(z)+S^{T} \hat{\alpha}(z, \theta)-S^{T} D u-S^{T} H_{1}(x, u, \Lambda) .
\end{aligned}
$$


Then, (25) can be rewritten as follows:

$$
\begin{aligned}
\frac{d}{d t}\left[\frac{1}{2} S^{T} G_{1}(x) S\right]= & -S^{T} \tilde{\theta}^{T} \psi(z)+S^{T} \hat{\alpha}(z, \theta)-S^{T} D u+S^{T} H_{2}(x, u, S) \\
& -\frac{1}{2} \sum_{i=1}^{p} \sigma_{\theta i}\left|S_{i}\right| \mid \theta_{i}^{*} \|^{2}
\end{aligned}
$$

with $H_{2}(x, u, S)=\varepsilon(z)-H_{1}(x, u, \Lambda)+\vartheta^{*} \operatorname{Sign}\left(S^{T}\right)$, with

$\vartheta^{*}=\operatorname{Diag}\left[0.5 \sigma_{\theta 1}|| \theta_{1}^{*}\left\|^{2}, 0.5 \sigma_{\theta 2}|| \theta_{2}^{*}\right\|^{2}, \ldots, 0.5 \sigma_{\theta p} \|\left.\theta_{p}^{*}\right|^{2}\right]$

$\sigma_{\theta i}$ (for $i=1, \ldots, p$ ) is a small positive constant that will be defined later.

In the sequel, one needs the following realistic assumption.

Assumption 4. One assumes that

$\left|H_{2}(x, u, S)\right| \leq \kappa^{*} \bar{H}(x, u)$

with $\bar{H}(x, u)=1+|| x||+|| u||$, where $\kappa^{*}=\left[\kappa_{1}^{*}, \kappa_{2}^{*}, \ldots, \kappa_{p}^{*}\right]^{T}$ is an unknown constant vector.

Remark 6. As started by [38], this assumption is not restrictive. In fact, $H_{1}(x, u, \Lambda)$ can be theoretically approximated over a large compact set by a first-order TS fuzzy system [1] as follows: $H_{1}(x, u, \Lambda)=\theta_{h}^{* T} \psi_{h}(x, u, \Lambda) Z+\varepsilon_{h}(x, u, \Lambda) \quad$ with $\quad \psi_{h}(x, u, \Lambda)$ is the FBF vector (Note that this approximation is only used for analysis purposes, because $\Lambda(t)$ is unknown), $\theta_{h}^{*}$ is a real constant matrix, $\varepsilon_{h}(x, u, d)$ is a bounded fuzzy approximation error, and $Z=\left[1, x^{T}, u^{T}, \Lambda^{T}\right]^{T}$. Since the matrix $\theta_{h}^{*}$ is constant, and $(\Lambda(t)$, $\psi_{h}(x, u, \Lambda)$ and $\left.\varepsilon_{h}(x, u, \Lambda)\right)$ are bounded, one can easily show that $\left|H_{1}(x, u, \Lambda)\right| \leq \mu^{*}(1+|| x||+|| u||)$, where $\mu^{*}$ is a given positive constant. Finally, since $\varepsilon(z)$ is bounded, then one can obtain (28).

For the system (1), one can consider the following fuzzy adaptive controller:

$u=N(\zeta)\left[-\hat{\alpha}(z, \theta)-K S-u_{r}\right]$

with

$N(\zeta)=\varsigma^{2} \cos (\zeta)$

$\dot{\zeta}=S^{T}\left[\hat{\alpha}(z, \theta)+K S+u_{r}\right]$,

and $K=\operatorname{Diag}\left[k_{1}, k_{2}, \ldots, k_{p}\right]$, where $k_{i}>0, i=1, \ldots, p$, are free positive design constants. Recall that the Nussbaum gain function $N(\zeta)$ is used here to estimate the true control direction. The dynamic robust control term $u_{r}=\left[u_{r 1}, \ldots, u_{r p}\right]^{T}$, will be given below, allows to dynamically compensate for the effect of the uncertain nonlinearity $\mathrm{H}_{2}(x, u, S)$.

After substituting the control law (29) into (26), one gets

$$
\begin{aligned}
\frac{d}{d t} & {\left[\frac{1}{2} S^{T} G_{1}(x) S\right] \leq-\sum_{i=1}^{p} \tilde{\theta}_{i}^{T} \psi_{i}\left(z_{i}\right) S_{i}-\sum_{i=1}^{p} k_{i} S_{i}^{2}-\sum_{i=1}^{p} S_{i} u_{r i} } \\
& +\bar{H}(x, u) \sum_{i=1}^{p}\left|S_{i}\right| \kappa_{i}^{*}+(1+d N(\zeta)) \dot{\zeta}-\frac{1}{2} \sum_{i=1}^{p} \sigma_{\theta i}\left|S_{i}\right||| \theta_{i}^{*}||^{2}
\end{aligned}
$$

with $d=D_{i i}$, for $i=1, \ldots, p$, where $D_{i i}$ are diagonal terms of $D$.

The dynamic robust control term $u_{r i}$ is given by

$\dot{u}_{r i}=-\gamma_{r i} u_{r i}+\gamma_{r i}\left[S_{i}-\frac{u_{r i}}{u_{r i}^{2}+\delta_{i}^{2}} \bar{H}(x, u) \kappa_{i}\left|S_{i}\right|\right]$, for $i=1, \ldots, p$

with

$\dot{\delta}_{i}=-\gamma_{\delta i} \frac{\delta_{i}}{u_{r i}^{2}+\delta_{i}^{2}} \bar{H}(x, u) \kappa_{i}\left|S_{i}\right|, \quad$ for $\quad i=1, \ldots, p$

where $\kappa_{i}$ is the estimate of $\kappa_{i}^{*}$ defined in (28), $\gamma_{r i}$ and $\gamma_{\delta i}$ are positive design parameters and $\delta_{i}(0)>0$.
Adaptation laws associated to the proposed controller (29) are given by:

$\dot{\theta}_{i}=-\sigma_{\theta i} \gamma_{\theta i}\left|S_{i}\right| \theta_{i}+\gamma_{\theta i} S_{i} \psi_{i}\left(z_{i}\right)$

$\dot{\kappa}_{i}=\gamma_{\kappa i} \bar{H}(x, u)\left|S_{i}\right|$

where $\gamma_{\theta i}, \gamma_{\kappa i}$ and $\sigma_{\theta i}$ are positive design constants.

Remark 7. Due to special structure of the nonlinearities $\alpha(z)$ (Eqs. (15) and (16)) and due to dynamical feature of the control robust compensator $u_{r}$ (given by Eqs. (33) and (34)), our adaptive controller is free of the algebraic loop.

Our main result is summarized by the following theorem.

Theorem 1. Consider the system (1) with Assumptions 1-4. Then, the control law defined by (29)-(31) and (33)-(36) can guarantee the following properties:

- All signals in the closed-loop system are bounded.

- The tracking errors and their derivatives asymptotically decrease to zero, i.e., $e_{i}^{(j)}(t) \rightarrow 0$ as $t \rightarrow \infty$ for $i=1, \ldots, p$ and $j=0,1, \ldots, r_{i}-1$.

Proof of Theorem 1. Let us consider the following Lyapunov function candidate:

$$
\begin{aligned}
V= & \frac{1}{2} S^{T} G_{1}(x) S+\frac{1}{2} \sum_{i=1}^{p} \frac{1}{\gamma_{\theta i}} \tilde{\theta}_{i}^{T} \tilde{\theta}_{i}+\frac{1}{2} \sum_{i=1}^{p} \frac{1}{\gamma_{k \mathrm{i}}} \tilde{\kappa}_{i}^{2}+\frac{1}{2} \sum_{i=1}^{p} \frac{1}{\gamma_{\delta i}} \delta_{i}^{2} \\
& +\frac{1}{2} \sum_{i=1}^{p} \frac{1}{\gamma_{\mathrm{ri}}} u_{r i}^{2}
\end{aligned}
$$

with $\tilde{\kappa}_{i}=\kappa_{i}-\kappa_{i}^{*}$, for $i=1, \ldots, p$.

The time derivative of $V$ can be given by:

$$
\begin{aligned}
\dot{V}= & S^{T} G_{1}(x) \dot{S}+\frac{1}{2} S^{T} \dot{G}_{1}(x) S+\sum_{i=1}^{p} \frac{1}{\gamma_{\theta \mathrm{i}}} \tilde{\theta}_{i}^{T} \dot{\theta}_{i}+\sum_{i=1}^{p} \frac{1}{\gamma_{k \mathrm{i}}} \tilde{\kappa}_{i}^{2} \dot{\kappa}_{i} \\
& +\sum_{i=1}^{p} \frac{1}{\gamma_{\delta i}} \delta_{i} \dot{\delta}_{i}+\sum_{i=1}^{p} \frac{1}{\gamma_{\mathrm{ri}}} u_{r i} \dot{u}_{r i}
\end{aligned}
$$

Using (32)-(36), (38) can be rewritten as follows:

$$
\begin{aligned}
\dot{V} & \leq-\sum_{i=1}^{p} k_{i} S_{i}^{2}-\sum_{i=1}^{p} u_{r i}^{2}+(1+d N(\zeta)) \dot{\zeta}-\sum_{i=1}^{p} \sigma_{\theta i}\left|S_{i}\right| \tilde{\theta}_{i}^{T} \theta_{i} \\
& -\left.\frac{1}{2} \sum_{i=1}^{p} \sigma_{\theta i}\left|S_{i}\right||| \theta_{i}^{*}\right|^{2}
\end{aligned}
$$

One can easily check that

$2\left|S_{i}\right| \tilde{\theta}_{i}^{T} \theta_{i} \geq\left|S_{i}\right||| \tilde{\theta}_{i}||^{2}-\left|S_{i}\right||| \theta_{i}^{*}||^{2}$,

using (40), $\dot{V}$ can be bounded as follows:

$\dot{V} \leq-\sum_{i=1}^{p} k_{i} S_{i}^{2}+(1+d N(\zeta)) \dot{\zeta}$

Integrating $(41)$ over $[0, t]$, one has

$V(t) \leq V(t)+\int_{0}^{t} \sum_{i=1}^{p} k_{i} S_{i}^{2} d \tau \leq V(0)+\int_{0}^{t}(\dot{\zeta}+d N(\zeta) \dot{\zeta}) d \tau$

According to Lemma 2, [13,50], one has $V(t), \int_{0}^{t}(1+d N(\zeta)) \dot{\zeta} d \tau$ and $\zeta$ which are bounded on $\left[0, t_{f}\right)$. Similar to discussion in $[13,50]$, one knows that the above discussion is also true for $t_{f}=+\infty$, therefore $S, \tilde{\theta}_{i}, \tilde{\kappa}_{i}, \delta_{i}$ and $u_{r i} \in L_{\infty}$. This implies the 
boundedness of $\theta_{i}, \quad \kappa_{i}, x$ and $u$. From (41) and since $\int_{0}^{\infty}(1+d N(\zeta)) \dot{\zeta} d \tau$ and $V(t)$ are bounded, it is easy to show that $\int_{0}^{\infty} \sum_{i=1}^{p} S_{i}^{2} d t$ exists, i.e., $S_{i} \in L_{2}$.

In order to show the boundedness of $\dot{S}_{i}$, one can rearrange Eq. (15) as follows:

$\dot{S}=G_{1}^{-1}(x)\left[-0.5 \dot{G}_{1}(x) S+\alpha(z)-D u-H_{1}(u, x, \Lambda)\right]$.

From (43), since $(x, S, u) \in L_{\infty}$ and $G_{1}(x)$ is a positive-definite matrix (i.e., $\exists \sigma_{0}>0$, such as ||$G_{1}(x)|| \geq \sigma_{0}$ ), and all functions in (43) are continuous, one can conclude about the boundedness of $\dot{S}_{i}$ (i.e., one has $\dot{S}_{i} \in L_{\infty}$ ).

Finally, since $S_{i} \in L_{2} \cap L_{\infty}$ and $\dot{S}_{i} \in L_{\infty}$, by using Barbalat's lemma one can conclude that $S_{i}(t) \rightarrow 0$ as $t \rightarrow \infty$. Therefore, the tracking errors and their derivatives converge asymptotically to zero, i.e., $e_{i}^{(j)}(t) \rightarrow 0$ as $t \rightarrow \infty$ for $i=1 \ldots p$ and $j=0,1, \ldots, r_{i}-1$.

Remark 8. The main contributions of this paper with respect to the existing works in the literature [32,11,13,40-42] are emphasized below.

a) Despite the large class of the nonaffine systems considered in $[40,42]$, the controllers proposed in $[40,42]$ cannot be applied to our system (1), because the latter has not a triangular structure form. Moreover, unlike this paper, in $[40,42]$ only a practical stability is guaranteed.

b) Although a similar class of MIMO nonaffine systems has been considered in [41], the fuzzy adaptive controller designed in [41] has not been derived rigorously in mathematics. In fact, the time derivative of the Lyapunov function candidate is completely false. Moreover, the sign of the control gain matrix is assumed to be known and the effect of the external disturbance has not been considered.

c) Unlike this paper, the design of a fuzzy adaptive outputfeedback control system for a simple class of affine SISO nonlinear systems with unknown control direction has been considered in [32].

d) Unlike this paper, in [11,13], a simple class of MIMO affine nonlinear systems with unknown control direction has been considered. Note that the extension of our results in $[11,13]$ to a perturbed nonaffine MIMO system (1) is not a trivial task because of the uncertain nonlinear HOT which depend on the input vector $u$. In this paper, for dealing with this problem, a dynamic adaptive robust control term has been introduced.

Remark 9. If the matrix $D$ obtained by the matrix factorization (10) is known, one does not need to use the Nussbaum function in the control law (29)-(31) and (33)-(36). In this case, the control law becomes:

$u=D\left[\hat{\alpha}(z, \theta)+K S+u_{r}\right]$

with

$\dot{u}_{r i}=-\gamma_{r i} u_{r i}+\gamma_{r i}\left[S_{i}-\frac{u_{r i}}{u_{r i}^{2}+\delta_{i}^{2}} \bar{H}(x, u) \kappa_{i}\left|S_{i}\right|\right]$, for $i=1, \ldots, p$

$\dot{\delta}_{i}=-\gamma_{\delta i} \frac{\delta_{i}}{u_{r i}^{2}+\delta_{i}^{2}} \bar{H}(x, u) \kappa_{i}\left|S_{i}\right|, \quad$ for $\quad i=1, \ldots, p$

$\dot{\theta}_{i}=-\sigma_{\theta i} \gamma_{\theta i}\left|S_{i}\right| \theta_{i}+\gamma_{\theta i} S_{i} \psi_{i}\left(z_{i}\right)$

$\dot{\kappa}_{i}=\gamma_{\kappa i} \bar{H}(x, u)\left|S_{i}\right|$

It is worth noting that the design constants are defined as in the first controller, and the proof of the stability associated to the controller (44)-(48) and the convergence of tracking errors towards zero is straightforward.

\section{Simulation results}

This section presents an illustrative simulation example which highlights the performance of the proposed adaptive fuzzy controller. The control problem of an academic MIMO nonaffine uncertain system is considered to this end. Its dynamic equations are given by:

$$
\left\{\begin{array}{l}
\dot{x}_{11}=x_{12} \\
\dot{x}_{12}=x_{11}^{2}+x_{12}^{2}+0.15 u_{1}^{3}+\left(2+\cos \left(x_{11}\right)\right) u_{1}-u_{2}+\Lambda_{1}(t) \\
\dot{x}_{21}=x_{22} \\
\dot{x}_{22}=x_{22}^{2}+x_{11}+x_{12}^{2}-0.5 u_{1}+\left(1+x_{21}^{2}\right) u_{2}^{3}+\left(2+\sin \left(x_{21}\right)\right) u_{2}+\Lambda_{2}(t) \\
y_{1}(t)=x_{11}(t), y_{2}(t)=x_{21}(t) .
\end{array}\right.
$$

where $x=\left[x_{11}, x_{12}, x_{21}, x_{22}\right]^{T}$ is the state vector of the system, $u_{1}$ and $u_{2}$ are the control inputs, and $y_{1}$ and $y_{2}$ are the system outputs. $\Lambda_{1}(t)$ and $\Lambda_{2}(t)$ are external disturbances which are assumed to be square waves having an amplitude \pm 1 with a period of $2 \pi(s)$. The control objective consists in allowing the system outputs $y_{1}$ and $y_{2}$ to, respectively, track the sinusoidal desired trajectories $y_{d 1}=\sin (t)$ and $y_{d 2}=\sin (t)$. The fuzzy system $\theta_{1}^{T} \psi_{1}\left(z_{1}\right)$ has the vector $\left[x^{T}, u_{2}\right]^{T}$ as input, while the fuzzy system $\theta_{2}^{T} \psi_{2}\left(z_{2}\right)$ has the state vector $x$ as input. For each variable of the entries of these fuzzy systems, as in [52], we define three (one triangular and two trapezoidal) membership functions uniformly distributed on the intervals $[-2,2]$ for $x_{11}, x_{12}, x_{21}$, and $x_{22}$, and $[-10,10]$ for $u_{2}$.

The design parameters are chosen as follows: $\gamma_{\theta 1}=600$, $\gamma_{\theta 2}=600, \sigma_{\theta 1}=\sigma_{\theta 2}=0,001, \gamma_{\kappa 1}=\gamma_{\kappa 2}=0.5, \gamma_{r 1}=\gamma_{r 2}=100, \gamma_{\delta 1}=$ $\gamma_{\delta 2}=0.005, \lambda_{1}=\lambda_{2}=2, k_{1}=k_{2}=1$. The initial conditions are selected as: $\quad x(0)=\left[\begin{array}{llll}0.5 & 0 & 0.5 & 0\end{array}\right], \quad u_{r 1}(0)=u_{r 2}(0)=0.1$, $\delta_{1}(0)=\delta_{2}(0)=1, \kappa(0)=0, \theta_{1}(0)=\theta_{2}(0)=0$, with 0 denotes the null vector. The simulation results are shown in Fig. 2 .

Fig. 2(a) and (b) illustrate the boundedness and convergence of the tracking errors for both subsystems. The boundedness of the corresponding control signals $\left(u_{1}, u_{2}\right)$ is well illustrated in Fig. 2(c). Fig. 2(d) also displays the control signals $\left(u_{1}, u_{2}\right)$ but for $t \in[0 s, 0.5 s]$.

Note that Fig. 2(d) shows that just one switching in the control direction was need. The jump made in the control signals at $t=0.13 \mathrm{~s}$, which also causes their amplification, is naturally due to the Nussbaum gain-function. After $t=0.13 s$, the control direction is correctly identified and all tracking errors vanish, but not in exponential way (see Fig. 2(a) and (b)). Finally, it is worth mentioning that the Nussbaum gain function-based control may generally have wild transient performance.

\section{Conclusion}

A novel fuzzy adaptive control algorithm for multivariable unknown nonaffine systems has been presented. In the controller designing, neither prior information about the control direction nor knowledge of the system nonlinearities are required. Indeed, adaptive fuzzy systems have been used to approximate unknown nonlinearities and a Nussbaum-type function has been particularly employed for dealing with the unknown control direction. Of fundamental interest, it has been shown that the underlying control system is stable and that the involved tracking errors converge to the origin. The effectiveness of the proposed controller is particularly emphasized throughout simulation results. 

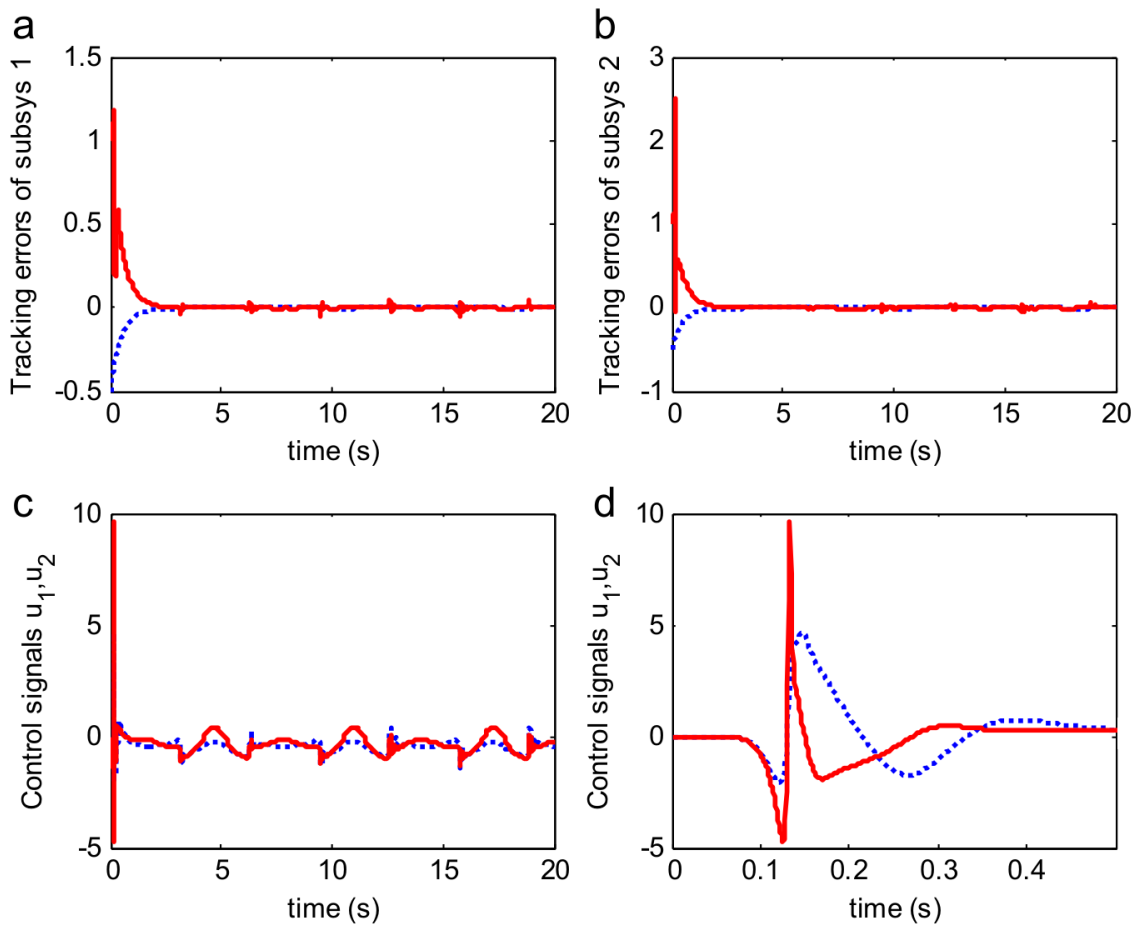

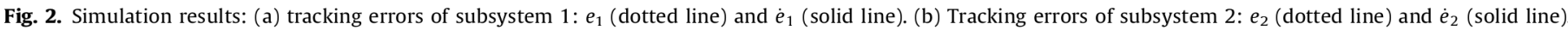
(c) Control input signals: $u_{1}$ (dotted line) and $u_{2}$ (solid line) (d). Control input signals for $t \in[0 s, 0.5 s]$ : $u_{1}$ (dotted line) and $u_{2}$ (solid line).

\section{References}

[1] L.X. Wang, Adaptive Fuzzy Systems and Control: Design and Stability Analysis, Prentice-Hall, Englewood Cliffs, NJ, 1994.

[2] S.C. Tong, J. Tang, T. Wang, Fuzzy adaptive control of multivariable nonlinear systems, Fuzzy Sets Syst. 111 (2) (2000) 153-167.

[3] S.C. Tong, H.X. Li, Fuzzy adaptive sliding model control for MIMO nonlinear systems, IEEE Trans. Fuzzy Syst. 11 (3) (2003) 354-360.

[4] S.C. Tong, B. Chen, Y. Wang, Fuzzy adaptive output feedback control for MIMO nonlinear systems, Fuzzy Sets Syst. 156 (2) (2005) 285-299.

[5] S. Labiod, M.S. Boucherit, T.M. Guerra, Adaptive fuzzy control of a class of MIMO nonlinear systems, Fuzzy Sets Syst. 151 (2005) 59-77.

[6] H.-X. Li, S.C. Tong, A hybrid adaptive fuzzy control for a class of nonlinear MIMO systems, IEEE Trans. Fuzzy Syst. 11 (1) (2003) 24-34.

[7] R. Ordonez, K.M. Passino, Stable multi-input multi-output adaptive fuzzy/ neural control, IEEE Trans. Fuzzy Syst. 7 (3) (1999) 345-353.

[8] Y.C. Chang, Robust tracking control for nonlinear MIMO systems via fuzzy approaches, Automatica 36 (2000) 1535-1545.

[9] H. Chekireb, M. Tadjine, D. Bouchaffra, Direct adaptive fuzzy control of nonlinear system class with applications, Control Intell. Syst. 31 (2) (2003) $1-11$.

[10] N. Essounbouli, A. Hamzaoui, J. Zaytoon, An improved robust adaptive fuzzy controller for MIMO systems, Control Intell. Syst. 34 (1) (2006) 12-21.

[11] A. Boulkroune, M. MSaad, H. Chekireb, Design of a fuzzy adaptive controller for MIMO nonlinear time-delay systems with unknown actuator nonlinearities and unknown control direction, Inf. Sci. 180 (2010) 5041-5059.

[12] A. Boulkroune, M. M'Saad, M. Tadjine, M. Farza, Adaptive Fuzzy Control for MIMO Nonlinear Systems with Unknown Dead-zone, in: Proc. of the 4th International IEEE Conference on Intelligent Systems, Varna, Bulgaria, pp. 450-455, Sep. 2008.

[13] A. Boulkroune, M. M'Saad, M. Tadjine, M. Farza, Fuzzy adaptive controller for MIMO nonlinear systems with known and unknown control direction, Fuzzy Sets Syst. 161 (2010) 797-820.

[14] A. Boulkroune, M. M'Saad, M. Farza, Adaptive fuzzy controller for multivariable nonlinear state time-varying delay systems subject to input nonlinearities, Fuzzy Sets Syst. 164 (2011) 45-65.

[15] S.S. Ge, C.C. Hang, T. Zhang, Nonlinear adaptive control using neural networks and its application to CSTR systems, J. Process Control 9 (1998) 313-323.

[16] M. Krstic, I. Kanellakopoulos, P.V. Kokotovic, Nonlinear and Adaptive Control Design, Wiley, New York, 1995.

[17] J.D. Boskovic, L. Chen, R.K. Mehra, Multivariable Adaptive Controller Design for a Class of Non-affine Models Arising in Flight Control, in: Proc. of the 40th IEEE Conference on Decision and Control, Orlando, Florida USA, pp. 24422447, December 2001
[18] A.S. Shiriaev, H. Ludvigsen, O. Egeland, A.L. Fradkov, Swinging up of Nonaffine in Control Pendulum, in: Proc. of American Control Conference, San Diego, California, USA, pp. 4039-4044, 1999.

[19] S.S. Ge, C.C. Hang, T. Zhang, Adaptive neural networks control of nonlinear systems by state and output feedback, IEEE Trans. Syst. Man Cybern. Part B Cybern. 29 (1999) 818-828.

[20] J.Y. Park, G.T. Park, Robust adaptive fuzzy controller for nonaffine nonlinear systems with dynamic rules activation, Int. J. Robust Nonlinear Control 13 (2003) 117-139.

[21] J.H. Park, S.H. Kim, Direct adaptive output feedback fuzzy controller for a nonaffine of nonlinear systems, IEEE Proc. Control Theory Appl. 151 (2004) 65-72.

[22] J.H. Park, S.H. Huh, S.H. Kim, S.J. Seo, G.T. Park, Direct adaptive controller for nonaffine nonlinear systems using self-structuring neural networks, IEEE Trans. Neural Networks 16 (2005) 414-422.

[23] S.S. Ge, C. Zhang, Adaptive NN control of uncertain nonlinear pure-feedback systems, Automatica 38 (2002) 1365-1372.

[24] T. Zhang, M. Guay, Adaptive control for a class of second-order nonlinear systems with unknown input nonlinearities, IEEE Trans. Syst. Man Cybern. Part B Cybern. 33 (2003) 143-149.

[25] S.S. Ge, J. Zhang, Neural-network control of nonaffine nonlinear system with zero dynamics by state and output feedback, IEEE Trans. Neural Networks 14 (2003) 900-918.

[26] H. Du, H. Shao, P. Yao, Adaptive neural network control for a class of lowtriangular-structured nonlinear systems, IEEE Trans. Neural Networks 17 (2006) 509-514.

[27] S. Labiod, T.M. Guerra, Adaptive fuzzy control for a class of SISO nonaffine nonlinear systems, Fuzzy Sets Syst. 158 (10) (2007) 1126-1137.

[28] Y.J. Liu, W. Wang, Adaptive fuzzy control for a class of uncertain nonaffine nonlinear systems, Inf. Sci. 177 (2007) 3901-3917.

[29] J.D. Boskovic, L. Chen, R. Mehra, Adaptive Tracking Control of a Class of Nonaffine Plants using Dynamic Feedback, in: Proc. of the 2001 American Control Conference, pp. 2450-2455, 2001.

[30] R. Boukezzoula, S. Galichet, L. Foulloy, Fuzzy Adaptive Linearizing Control for Non-affine Systems, in: Proc. of the IEEE International Fuzzy Systems Conference, pp. 543-548, 2003.

[31] N. Essounbouli, A. Hamzaoui, Direct and indirect robust adaptive fuzzy controllers for a class of nonlinear systems, Int. J. Control, Autom. Syst. 4 (2006) 146-154.

[32] A. Boulkroune, M. M'saad, On the Design of Observer-based Fuzzy Adaptive Controller for Nonlinear Systems with Unknown Control Gain Sign, Fuzzy Sets and Systems, 〈http://dx.doi.org/10.1016/j.fss.2011.12.005〉, Dec 2011.

[33] Y. Li, S.C. Tong, Adaptive fuzzy backstepping output feedback control of nonlinear systems with unknown virtual control coefficients using MT-filters, Neurocomputing 74 (2011) 1557-1563. 
[34] J. Kaloust, Z. Qu, Continuous robust control design for nonlinear uncertain systems without a priori knowledge of control direction, IEEE Trans. Autom. Control 40 (1995) 276-282.

[35] B. Brogliato, R. Lozano, Adaptive control of first-order nonlinear systems with reduced knowledge of the plant parameters, IEEE Trans. Autom. Control 39 (1994) 1764-1768.

[36] W.H. Dong, X.X. Sun, Y. Lin, Variable structure model reference adaptive control with unknown high frequency gain sign, Acta Autom. Sin. 33 (2007) 404-408.

[37] J.-H. Park, S.-H. Kim, C.-J. Moon, Adaptive fuzzy controller for the nonlinear system with unknown sign of the input gain, Int. J. Control, Autom. Syst. 4 (2) (2006) 178-186.

[38] S. Labiod, T.M. Guerra, Indirect adaptive fuzzy control for a class of nonaffine nonlinear systems with unknown control directions, Int. J. Control, Autom. Syst. 8 (4) (2010) 903-907.

[39] H.E. Psillakis, Further results on the use of Nussbaum gains in adaptive neural network control, IEEE Trans. Autom. Control 55 (12) (2010) 2841-2846.

[40] Y.J. Liu, W. Wang, Adaptive fuzzy control for a class of uncertain non-affine nonlinear systems, Inf. Sci. 177 (2007) 3901-3917.

[41] S. Doudou, F. Khaber, Direct adaptive fuzzy control of a class of MIMO nonaffine nonlinear systems, Int. J. Syst. Sci. 43 (6) (2012) 1029-1038.

[42] Y.J. Liu, S. Tong, W. Wang, Adaptive fuzzy output tracking control for a class of uncertain nonlinear systems, Fuzzy Sets Syst. 160 (2009) 2727-2754.

[43] J. Chen, A. Behal, D.M. Dawson, Adaptive Output Feedback Control for a Class of MIMO Nonlinear Systems, in: Proc. of the American Control Conference, Minneapolis, MN, pp. 5300-5305, June 2006.

[44] R.R. Costa, Li. Hsu, A.K. Imai, P. Kokotovic, Lyapunov-based adaptive control of MIMO systems, Automatica 39 (7) (2003) 1251-1257.

[45] L. Hsu, R.R. Costa, F. Lizarralde, Luapunov/passivity-based adaptive control of relative degree two MIMO systems with an application to visual servoing, IEEE Trans. Autom. Control 52 (2) (2007) 364-371.

[46] X.T. Zhang, D.M. Dawson, M.S. de Queiroz, B. Xian, Adaptive Control for a Class of MIMO Nonlinear Systems with Non-symmetric Input Matrix, in: Proc. of the IEEE International Conference on Control Applications, Taipei, Taiwan, pp. 1324-1329, Sep 2004.

[47] S.S. Ge, J. Wang, Robust adaptive neural control for a class of perturbed strict feedback nonlinear systems, IEEE Trans. Neural Networks 13 (6) (2002) 1409-1419.

[48] R.D. Nussbaum, Some remarks on the conjecture in parameter adaptive control, Syst. Control Lett. 1 (3) (1983) 243-246.

[49] T.P. Zhang, S.S. Ge, Adaptive neural control of MIMO nonlinear state timevarying delay systems with unknown dead-zones and gain signs, Automatica 43 (6) (2007) 1021-1033.

[50] T.P. Zhang, Y. YI, Adaptive fuzzy control for a class of MIMO nonlinea systems with unknown dead-zones, Acta Autom. Sin. 33 (1) (2007) 96-99.

[51] A. Boulkroune, M. Tadjine, M. M'Saad, M. Farza, General adaptive observerbased fuzzy control of uncertain nonaffine systems, Arch. Control Sci. 16(LII) (4) (2006) 363-390.

[52] A. Boulkroune, M. Tadjine, M. M'Saad, M. Farza, How to design a fuzzy adaptive controller based on observers for uncertain affine nonlinear systems, Fuzzy Sets Syst. 159 (2008) 926-948.

[53] A. Boulkroune, M. Tadjine, M. M'Saad, M. Farza, Adaptive fuzzy controller for non-affine systems with zero dynamics, Int. J. Syst. Sci. 40 (4) (2009) 367-382.

[54] S.C. Tong, H.X. Li, W. Wang, Observer-based adaptive fuzzy control for SISO nonlinear systems, Fuzzy Sets Syst. 148 (2004) 355-376.

[55] S.C. Tong, Y.M. Li, Observer-based fuzzy adaptive control for strict-feedback nonlinear systems, Fuzzy Sets Syst. 160 (2009) 1749-1764.

[56] W.Y. Wang, Y.H. Chien, Y.G. Leu, T.T. Lee, Adaptive T-S fuzzy-neural modeling and control for general MIMO unknown nonaffine nonlinear systems using projection update laws, Automatica 46 (2010) 852-863.

[57] H.G. Zhang, Y.B. Quan, Modeling, identification, and control of a class of nonlinear systems, IEEE Trans. Fuzzy Syst. 9 (2) (2001) 349-354.
[58] Z.Z. Mao, X.S. Xiao, Decentralized adaptive tracking control of nonaffine nonlinear large-scale systems with time delays, Inf. Sci. 181 (23) (2011) 5291-5303. 